\title{
Ophthalmic Pseudomonas infection in infancy
}

\author{
E M Boyle, J R Ainsworth, A V Levin, A N Campbell, M Watkinson
}

\begin{abstract}
Four infants developed invasive Pseudomonas aeruginosa ophthalmic infections between 5 and 90 days of age. Three died from septicaemia, and the fourth required enucleation of one eye. Absent red reflexes or other eye signs in a septicaemic infant merit urgent ophthalmological assessment for endophthalmitis, in particular, Pseudomonas.

(Arch Dis Child Fetal Neonatal Ed 2001;85:F139-F140)
\end{abstract}

Keywords: Pseudomonas aeruginosa; eye; endophthalmitis; bacteria; infection

Invasive eye infections are rare in neonates but occur in the presence of multisystem disease with a high mortality. ${ }^{1-3}$

\section{Case reports}

Infant 1 was born at 29 weeks after six days prolonged rupture of the membranes (PROM). At birth he needed ventilation, cardiac massage, and adrenaline. He was ventilated for respiratory distress syndrome. Gentamicin and benzylpenicillin were given, and therapeutic levels of gentamicin were achieved. Initial swabs and blood culture were negative, but on day 3 Pseudomonas aeruginosa, sensitive to gentamicin, was isolated from airway secretions and eye discharge. He improved and was extubated. On day 7 he appeared septicaemic. Red reflexes were absent, and a white ring was visible around each pupil. Purulent vitreous fluid was aspirated and he was treated with intravitreal and intravenous ceftazidime. $\mathrm{He}$ died the same day. $P$ aeruginosa was cultured from the vitreous fluid, and the lungs and heart at post mortem.

Infant 2 was born at 33 weeks with gastroschisis which was repaired in the neonatal period. He remained in hospital pending further surgery. At 3 months, he developed abdominal distension, respiratory distress, and corneal clouding. He required ventilation and inotropic support despite intravenous piperacillin, ticarcillin, and gentamicin, followed by ceftazidime, tobramycin, and vancomycin. $P$ aeruginosa was grown from blood cultures, the central venous catheter, endotracheal aspirates, and pustular skin lesions. It was sensitive to all of the antibiotics in use except tobramycin and vancomycin. Two days later, a white ring was visible around each pupil (fig 1), and the red reflexes were absent. Vitreous aspiration was performed with intravitreal injection of vancomycin and gentamicin, and subconjunctival injection of ceftazidime and gentamicin. $P$ aeruginosa was cultured from the aspirates. $\mathrm{He}$ died the next day. Autopsy showed culture positive bilateral pan-endophthalmitis, leptomeningitis, bronchopneumonia, and ecthyma gangrenosum.

Infant 3 was a previously well 6 week old girl with a discharge from her red eye for two days. Her fontanelle was tense. A profound apnoea necessitated ventilation. The right eye showed proptosis, absent red reflex, corneal clouding, but no hypopyon. She was thought to have a conjunctival/corneal infection. Gentamicin eye drops and intravenous cefotaxime were started. After 24 hours, $P$ aeruginosa was cultured from blood and cerebrospinal fluid. Treatment was immediately changed to ceftazidime and gentamicin. She died that day. Autopsy was declined.

Infant 4 was born at 30 weeks after 3 days PROM. She was well, but received intravenous benzylpenicillin and gentamicin for 48 hours. Initial cultures were negative, although $P$ aeruginosa was grown from a maternal high vaginal swab. On day 5 she developed crusting around the right eye. A conjunctival swab, which later grew $P$ aeruginosa, was obtained, and chloramphenicol drops instilled. Within 24 hours, a central corneal perforation developed. Ciprofloxacin eye drops and intravenous gentamicin were started, but the perforation enlarged. Later the lens protruded and the ocular contents were lost. Two years later she had had no further problems and wore a cosmetically acceptable artificial eye.

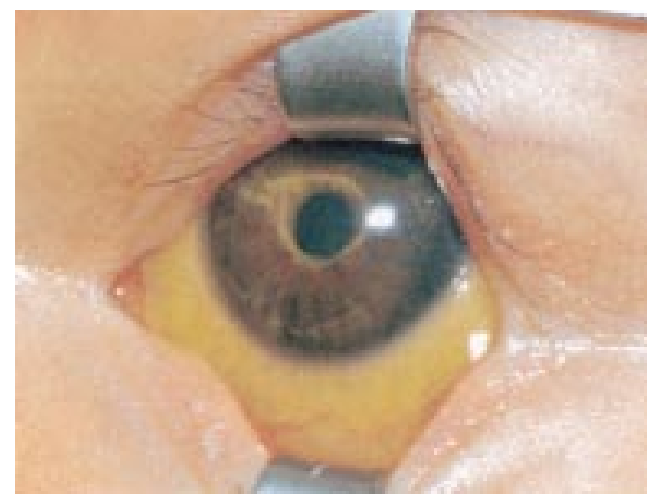

Figure 1 Pseudomonas aeruginosa endophthalmitis in a neonate, showing a whitish ring around the pupil consistent with hypopyon material lying on the iris in the supine patient. 
Discussion

Endogenous endophthalmitis results from haematogenous spread to the eye secondary to septicaemia. In exogenous endophthalmitis, the infection develops initially in the eye as a result of corneal infection, perforating injury, or intraocular surgery. Infant 4 had exogenous endophthalmitis whereas in the others it was endogenous. P aeruginosa has been identified as a causative organism in more than $75 \%$ of invasive neonatal eye infections. ${ }^{1}$ It was not isolated from the discharge of the patient in case 3, but we feel that the corneal infection and orbital cellulitis were almost certainly pseudomonal. An association between pseudomonal conjunctivitis and meningitis has been reported. ${ }^{4}$ Although the neonatal unit is a high risk area for nosocomial infection, three of our patients were infected before admission. Infant 3 was infected on admission, and infants 1 and 4 had PROM, with a positive high vaginal swab in one. Two babies received intravenous gentamicin for at least 48 hours from birth, but still developed infections. This may reflect the virulence of the bacterium. ${ }^{5}$

Diagnosis is confirmed by microscopy and culture of aspirated vitreous fluid. Culture of the purulent discharge is useful but less reliable in identifying the causative organism, being found in only two of our four cases. Nevertheless, growth of $P$ aeruginos a from discharge from an eye in a sick child should alert the clinician to the risk of a sight threatening and life threatening illness.

The most appropriate treatment for endophthalmitis is a combination of intravenous cephalosporin and aminoglycoside, ${ }^{6}$ - for example, ceftazidime $50 \mathrm{mg} / \mathrm{kg}$ every 12 hours and gentamicin $4 \mathrm{mg} / \mathrm{kg}$ every 24 hours. As intraocular accumulation of intravenous antibiotics is poor, the use of intravitreal antibiotics is

\section{Key messages}

- Loss of red reflex, a change in the appearance of the iris, or purulent eye discharge can be clues to a life threatening ocular and systemic infection

- Bilateral endophthalmitis can complicate neonatal pseudomonal septicaemia, and is a grave prognostic sign for survival

- Treatment of neonatal endophthalmitis should include intravitreal and intravenous antibiotics

essential - for example, ceftazidime $2.25 \mathrm{mg}$ in $0.1 \mathrm{ml}^{6}$. Topical treatment may be used, but not as sole treatment.

Morbidity and mortality in pseudomonal endophthalmitis are high despite early diagno$\operatorname{sis}^{12}$; the deaths of three of our four infants reflect this. For survivors, the usual result is blindness of the affected eye, ${ }^{13}$ with enucleation sometimes being necessary.

We are grateful to Dr H P Roper and Dr S J Rose for permission to report cases under their care.

1 Lohrer R, Belohradsky BH. Bacterial endophthalmitis in neonates. Eur F Pediatr 1987;146:354-9.

2 Rais-Bahrami K, Platt P, Naqui M. Neonatal pseudomonas sepsis: even early diagnosis is too late. Clin Pediatr 1990;29:444

3 Schutze GE, Englund JA, Bresee JS. Pseudomonas aeruginosa endogenous endophthalmitis in a neonate. Pediatr Infect Dis 7 1989;8:893-4.

4 Shah SS, Gloor P, Gallagher PG. Bacteraemia, meningitis and brain abscess in a hospitalized infant: complications of Pseudomonas aeruginosa conjunctivitis. $\mathcal{f}$ Perinatol 1999;19:462-5.

5 Wasserman BN, Sondhi N, Carr BL. Pseudomonas-induced bilateral endophthalmitis with corneal perforation in a neonate. F AAPOS 1999;3:183-4.

6 Alio JL, Munoz G. Infectious endophthalmitis. In: BenEzra $\mathrm{D}$, ed. Ocular inflammation: basic and clinical concepts. London: Martin Dunitz, 1999:275-96. 